\title{
OPTIMAL TIME-FREE NODAL TRANSFERS BETWEEN ELLIPTICAL ORBITS $\dagger$
}

\author{
NguYen X. Vinh and Shau Hern Kuo \\ and \\ Christian Marchal \\ ONERA, 92322 Châtillon, France
}

The University of Michigan, Ann Arbor, MI 48109-2140, U.S.A.

(Received 24 February 1988)

\begin{abstract}
This paper presents the necessary conditions for the minimum fuel, time-free transfer between two non-coplanar elliptical orbits. It is shown that the solution is obtained by solving a system of three non-linear equations for three unknowns. In the second part, we discuss the case where the impulses are applied along the line of nodes. In general, this nodal transfer is non optimal, but the characteristic velocity for the best nodal transfer, called the minimizing nodal transfer, is reasonably close to the one for the optimal transfer for it to be useful as a substitute for a practical transfer. Furthermore, when we continuously vary the relative position of the two terminal orbits, the two characteristic velocities, for the minimizing nodal transfer and the optimal transfer, exhibit the same trend in the sense that they pass through their maxima and minima at nearly the same relative position. This makes the set of explicit formulas for computing the minimizing nodal transfer, as presented in this paper, a useful tool for designing a minimum fuel transfer between several orbits.
\end{abstract}

\section{INTRODUCTION}

With the advent of the orbital transfer vehicle, there is a current research interest in the analysis of combined aerodynamic and propulsive maneuver as a technique for reduction of the fuel consumption in orbital transfer as compared to the pure propulsive maneuver. In the published literature, these comparisons are usually made in simple cases such as the transfer between non-coplanar circular orbits. This is because the computation of the optimal propulsive transfer between non-coplanar elliptical orbits in itself is a difficult problem.

In this paper we shall first present the necessary conditions for the minimum fuel, time-free, twoimpulse transfer between two arbitrary ellipitical orbits. It is shown that basically the solution is obtained by solving a system of three non-linear equations for three unknowns. In the second part, we restrict the discussion of the optimal solution to the case of nodal transfers, that is the transfers where all the impulses are applied along the line of nodes. In general, they are non-optimal. They are possibly optimal only in the one-impulse or the two-impulse cases, except when the terminal orbits are coaxial the three-impulse optimal transfer also exists. When the terminal orbits are non-coaxial, it is easy to compute the one-impulse transfer when the orbits are intersecting, and also the best two-impulse transfer in the general case of non intersecting orbits. Then, this transfer, called the minimizing nodal transfer, is truly

$\uparrow$ Paper IAF-87-325 presented at the 38th Congress of the International Astronautical Federation, Brighton, U.K., 10-17 October 1987. optimal only when some inequalities are verified, and also when one additional equality is satisfied in the two-impulse case.

In practical applications, the cost for the minimizing nodal transfer is close to the optimal solution. Hence, the best nodal transfer can be used as a practical transfer between non-coplanar elliptical orbits. It also serves as a reasonable upper bound for the fuel consumption in the search of the true optimal transfer.

\section{NECESSARY CONDITIONS}

Consider a two-impulse, time-free transfer between an initial orbit $\mathrm{O}_{1}$ and a final orbit $\mathrm{O}_{2}$. The two orbits are connected by a transfer orbit $\mathrm{O}$ through the applications of the impulses $I_{1}$ and $I_{2}$ (Fig. 1). Along the transfer orbit with center of attraction at point $F$, let MSTW be a rotating coordinate system with the origin $\mathrm{M}$ at the vehicle, the $\mathrm{S}$-axis along the position vector positive outwards, the $\mathrm{T}$-axis along the circumferential direction in the plane of $\mathrm{O}$, positive toward the direction of motion and the $\mathrm{W}$-axis completing a right-handed system. Then, if $\mathrm{S}, \mathrm{T}$, and $\mathrm{W}$ are the direction cosines of the optimal impulses, we have the identities

$$
\mathrm{S}_{i}^{2}+\mathrm{T}_{i}^{2}+\mathrm{W}_{i}^{2}=1, \quad i=1,2 .
$$

It is convenient to define the optimal direction of the impulse by its azimuth $\psi$ and elevation $\phi$. Then at the impulses

$$
\begin{aligned}
\mathrm{S}_{i} & =\sin \phi_{i}, \mathrm{~T}_{i}=\cos \phi_{i} \cos \psi_{i}, \mathrm{~W}_{i}=\cos \phi_{i} \sin \psi_{i} \\
i & =1,2
\end{aligned}
$$




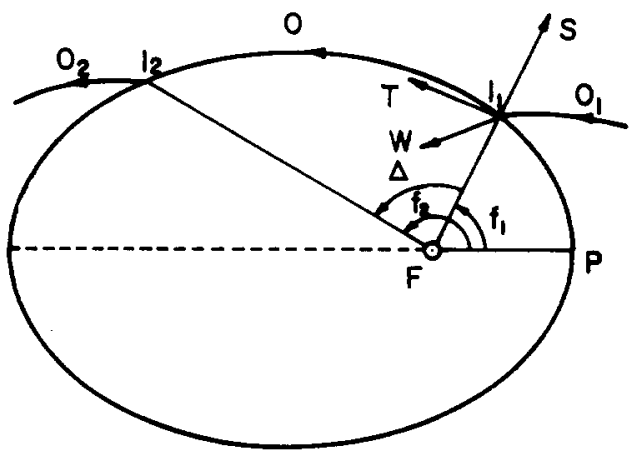

Fig. 1. Rotating coordinates and transfer geometry.

Let $f_{i}$ be the true anomalies of the points of application of the impulses, measured on the transfer orbit with eccentricity $e$. The transfer angle is

$$
\Delta=f_{2}-f_{1},
$$

From Lawden's theory of primer vector, it can be shown that for the transfer to be optimal, we have three necessary conditions, called the optimal switching conditions, relating the elements $e, f_{i}, \mathrm{~S}_{i}, \mathrm{~T}_{i}$ and $\mathrm{W}_{i}[1-3]$.

$$
\begin{gathered}
\left(1+q_{1}\right) J_{1}-q_{1} \mathrm{~T}_{1}=\left(1+q_{2}\right) J_{2}-q_{2} \mathrm{~T}_{2} \\
\left(y_{1} \mathrm{~T}_{1}-q_{1} \mathrm{~S}_{1}\right)\left(\mathrm{T}_{1}-J_{1}\right) \\
-\mathrm{S}_{1} \mathrm{~T}_{1}+y_{1} \mathrm{~W}_{1}^{2}+K_{1} \mathrm{~W}_{1}=0 \\
\left(y_{2} \mathrm{~T}_{2}-q_{2} \mathrm{~S}_{2}\right)\left(T_{2}-J_{2}\right) \\
-\mathrm{S}_{2} \mathrm{~T}_{2}+y_{2} \mathrm{~W}_{2}^{2}+K_{2} \mathrm{~W}_{2}=0 .
\end{gathered}
$$

In the following, we shall use the notation

$$
\begin{aligned}
& x=e \cos f, \quad y=e \sin f, \quad q=1+e \cos f, \\
& J_{1}=\left(\mathrm{S}_{2}-\mathrm{S}_{1} \cos \Delta\right) / \sin \Delta, \\
& J_{2}=\left(\mathrm{S}_{2} \cos \Delta-\mathrm{S}_{1}\right) / \sin \Delta, \\
& K_{1}=\left(q_{2} \mathrm{~W}_{2}-q_{1} \mathrm{~W}_{1} \cos \Delta\right) / \sin \Delta, \\
& K_{2}=\left(q_{2} W_{2} \cos \Delta-q_{1} W_{1}\right) / \sin \Delta .
\end{aligned}
$$

In addition to these three switching equations, some additional conditions and relations are required. First, for the impulse $I_{1}$, to precede the impulse $I_{2}$ in an optimal way, it is necessary that [3]

$$
q_{1} \mathrm{~T}_{1} \geqslant q_{2} \mathrm{~T}_{2} \text {. }
$$

Furthermore, for the case where the transfer orbit is completely outside the attracting planet, we must verify the condition that the magnitude of the primer vector along all the three orbits involved does not exceed unity. Along the transfer orbit $\mathrm{O}$, this condition is[3]

$$
A_{3} \geqslant \sqrt{A_{1}^{2}+A_{2}^{2}}=2 e\left(\mathrm{~S}_{1}^{2}+J_{1}^{2}\right)
$$

where

$$
\begin{aligned}
& A_{1}=2 e\left(\mathrm{~S}_{1} \mathrm{~S}_{2}-J_{1} J_{2}\right), \\
& A_{2}=2 e\left(\mathrm{~S}_{1} J_{2}+\mathrm{S}_{2} J_{1}\right),
\end{aligned}
$$

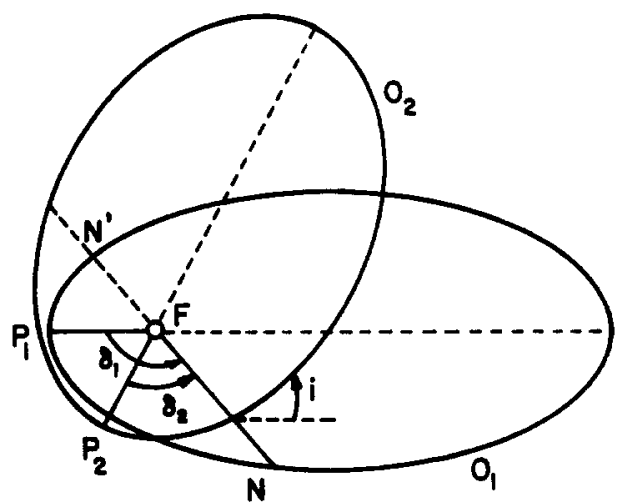

Fig. 2. Geometry of the terminal orbits.

$$
\begin{aligned}
A_{3}= & -A_{1} \cos f_{1}-A_{2} \sin f_{1} \\
& +2\left[q_{1}\left(1-3 \mathrm{~S}_{1}^{2}\right)-B^{2}\right] /(1-\cos \Delta), \\
B^{2}= & q_{1}^{2}\left(\mathrm{~T}_{1}-J_{1}\right)^{2}+\left[y_{1}\left(\mathrm{~T}_{1}-J_{1}\right)+\mathrm{S}_{1}\right]^{2} \\
& +\left[\left(\mathrm{T}_{1}-J_{1}\right)\left(y_{1} \mathrm{~T}_{1}-q_{1} \mathrm{~S}_{1}\right)-\mathrm{S}_{1} \mathrm{~T}_{1}\right]^{2} / \mathrm{W}_{1}^{2} .
\end{aligned}
$$

The condition (9) always implies

$$
\mathrm{S}_{1}^{2}+J_{1}^{2}=\mathrm{S}_{2}^{2}+J_{2}^{2} \leqslant \frac{1}{4} \text {. }
$$

This restricts the elevation angle of the optimal impulse to be always less than $30^{\circ}$ from the local horizontal plane.

To find the solution for a transfer by two impulses between given terminal orbits $\mathrm{O}_{1}$ and $\mathrm{O}_{2}$, we need the relations connecting these orbits with the transfer orbit $\mathrm{O}$ and the expressions for the impulses. The geometry of the terminal orbits is defined by their eccentricities $e_{1}$ and $e_{2}$, semi-latus recta $p_{1}$ and $p_{2}$, the angle $i$ between the orbital planes which intersect each other along the line of nodes FN, and the two angles $\delta_{1}$ and $\delta_{2}$ which are the true anomalies of this line of node in the initial and final plane respectively (Fig. 2). We acknowledge that in celestial mechanics if the plane of $\mathrm{O}_{1}$ is used as the reference plane and the direction $F P_{1}$ to its perigee is used as the reference direction, then the angular orientation of the orbit $\mathrm{O}_{2}$ is defined by the longitude of the ascending node $\Omega_{2}$, the inclination $i_{2}$ and the argument of the perigee $\omega_{2}$. Here we use $i, \delta_{1}$ and $\delta_{2}$ for the sake of symmetry in the resulting equations with the obvious relations $i=i_{2}, \delta_{1}=\Omega_{2}, \delta_{2}=2 \pi-\omega_{2}$. To solve the general problem of optimal two-impulse non-coplanar transfer between elliptical orbits, it has been suggested in[4] to use the unknowns: $p$ for the semi-latus rectum of the transfer orbit, and $\theta_{1}$ and $\theta_{2}$ to be respectively, the true anomalies of the points of application of the impulse on the initial and the final orbit. All the pertinent elements can be expressed in terms of these variables and the given data $e_{i}, p_{i}, \delta_{i}$ and $i$.

Let $r_{i}$ be the radial distance to the impulse $\mathrm{I}_{i}$. Then

$$
r_{i}=p_{i} /\left(1+e_{i} \cos \theta_{i}\right) .
$$

Next, in the unit sphere, after the application of the impulse $I_{1}$, the plane $O_{1}$ is rotated to the transfer 


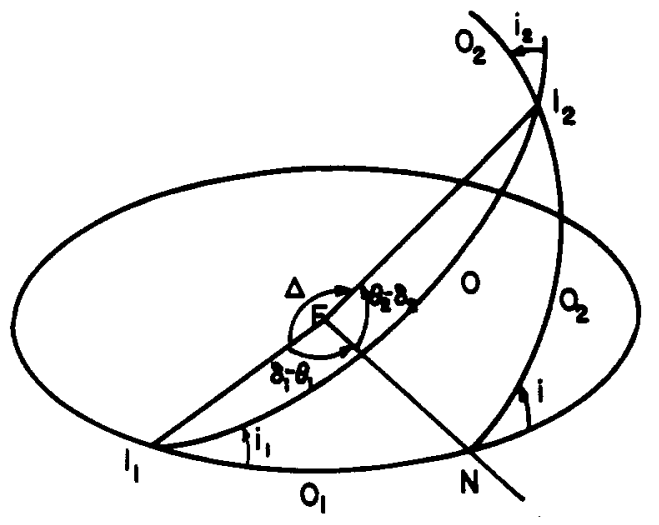

Fig. 3. Spherical geometry of the orbital planes.

plane $\mathrm{O}$ by the angle $i_{1}$ and after the application of the impulse $I_{2}$ the plane $O$ is rotated to the final plane $\mathrm{O}_{2}$ by the angle $i_{2}$ with the total plane change $i$ as prescribed (Fig. 3). From spherical trigonometry based on the figure with the angles as labeled, we have

$$
\begin{aligned}
\cos \Delta= & \cos \left(\delta_{1}-\theta_{1}\right) \cos \left(\delta_{2}-\theta_{2}\right) \\
& +\sin \left(\delta_{1}-\theta_{1}\right) \sin \left(\delta_{2}-\theta_{2}\right) \cos i, \\
\sin i_{1}= & -\frac{\sin \left(\delta_{2}-\theta_{2}\right)}{\sin \Delta} \sin i, \\
\sin i_{2}= & \frac{\sin \left(\delta_{1}-\theta_{1}\right)}{\sin \Delta} \sin i .
\end{aligned}
$$

Hence, $\Delta$ and $i_{i}$ are obtained explicitly in terms of $\theta_{i}$. In the figure, the two rotations, $i_{1}$ and $i_{2}$ are in the same direction. This is the plane change of the rotating type. In the case where $i_{2}$ is in the opposite direction, we have a plane change of the reflecting type. The same equations apply if we take a negative value for $i_{2}$. The true anomalies $f_{i}$ of the impulses $\mathrm{I}_{i}$ as measured in the transfer orbit are given by the equations

$$
\begin{aligned}
& \tan f_{1}=\cot \Delta-\frac{r_{1}\left(p-r_{2}\right)}{r_{2}\left(p-r_{1}\right) \sin \Delta}, \\
& \tan f_{2}=-\cot \Delta+\frac{r_{2}\left(p-r_{1}\right)}{r_{1}\left(p-r_{2}\right) \sin \Delta},
\end{aligned}
$$

which involve $p$ in addition to $\theta_{1}$ and $\theta_{2}$. Finally the eccentricity of the transfer orbit is given by

$$
e=\frac{r_{2}-r_{1}}{r_{1} \cos f_{1}-r_{2} \cos f_{2}}
$$

Let $\Delta V_{i}$ be the impulsive velocity changes with components on the STW system being $u_{i}, v_{i}$, and $w_{i}$. Then if $\mu$ is the gravitational constant, we have

$$
\begin{aligned}
& u_{1}=\sqrt{\mu}\left(\frac{e}{\sqrt{p}} \sin f_{1}-\frac{e_{1}}{\sqrt{p_{1}}} \sin \theta_{1}\right), \\
& v_{1}=\frac{\sqrt{\mu}}{r_{1}}\left(\sqrt{p}-\sqrt{p_{1}} \cos i_{1}\right), \\
& w_{1}=\frac{\sqrt{\mu p_{1}}}{r_{1}} \sin i_{1},
\end{aligned}
$$

and

$$
\begin{aligned}
& u_{2}=\sqrt{\mu}\left(\frac{e_{2}}{\sqrt{p_{2}}} \sin \theta_{2}-\frac{e}{\sqrt{p}} \sin f_{2}\right), \\
& v_{2}=\frac{\sqrt{\mu}}{r_{2}}\left(\sqrt{p_{2}} \cos i_{2}-\sqrt{p}\right), \\
& w_{2}=\frac{\sqrt{\mu p_{2}}}{r_{2}} \sin i_{2} .
\end{aligned}
$$

The magnitude of the respective characteristic velocity is

$$
\Delta V_{i}=\left(u_{i}^{2}+v_{i}^{2}+w_{i}^{2}\right)^{1 / 2}
$$

We now summarize the problem. Two terminal orbits $\mathrm{O}_{1}$ and $\mathrm{O}_{2}$ are given. They are defined by the elements $e_{i}, p_{i}, \delta_{i}$ and the angle $i$ between their planes. To find the optimum transfer orbit $O$ connecting the given orbits, we use the three unknowns $p, \theta_{1}$, and $\theta_{2}$ which are the semi-latus rectum of the transfer orbit and the true anomalies of the impulses measured in the initial and the final orbit, respectively. The three non-linear equations to be solved are the switching eqns (4), (5) and (6). In these equations, all the elements $\Delta, x_{i}=e \cos f_{i}, y_{i}=e \sin f_{i}$ can be expressed in terms of the unknowns $p, \theta_{1}$, and $\theta_{2}$, while the direction cosines of the thrust (and hence the thrust angles themselves) are obtained from

$$
\mathrm{S}_{i}=\frac{u_{i}}{\Delta V_{i}}, \quad \mathrm{~T}_{i}=\frac{v_{i}}{\Delta V_{i}}, \quad \mathrm{~W}_{i}=\frac{w_{i}}{\Delta V_{i}} .
$$

From eqns (17) and (18), these direction cosines are also expressible in terms of the selected unknowns. Once the solution is obtained, the two conditions (8) and (9) must be verified to insure the optimality of the transfer.

\section{THE OPTIMAL NODAL TRANSFER}

We now impose the condition that the impulses are applied on the line of nodes. Then

$$
\Delta=f_{2}-f_{1}=\pi \text {. }
$$

From the definition (7), we obtain

$$
x_{1}+x_{2}=0, \quad y_{1}+y_{2}=0, \quad q_{1}+q_{2}=2 .
$$

From the same definition, we write

$$
\begin{aligned}
& J_{1}=\frac{\left(S_{1}+S_{2}\right)}{\sin \Delta}-S_{1} \cot \frac{\Delta}{2}, \\
& J_{2}=S_{2} \cot \frac{\Delta}{2}-\frac{\left(S_{1}+S_{2}\right)}{\sin \Delta},
\end{aligned}
$$

When $\Delta$ tends to $\pi$, we have

$$
\lim J_{1}=\lim \frac{\left(\mathbf{S}_{1}+\mathbf{S}_{2}\right)}{\sin \Delta}=-\lim J_{2}=J .
$$

With this, we write the switching relation (4)

$$
4 J=q_{1} \mathrm{~T}_{1}-q_{2} \mathrm{~T}_{2} \text {. }
$$


We deduce that $J$ is finite and as a consequence

$$
S_{1}+S_{2}=0 \text {. }
$$

Again, from the definition (7), we have singularity in $K_{1}$ and $K_{2}$. But now that $J_{1}$ and $J_{2}$ are finite, we see from eqns (5) and (6) that $K_{1}$ and $K_{2}$ are also finite. We write

$$
\begin{aligned}
& K_{1}=\frac{\left(q_{1} \mathrm{~W}_{1}+q_{2} \mathrm{~W}_{2}\right)}{\sin \Delta}-q_{1} \mathrm{~W}_{1} \cot \frac{\Delta}{2} \\
& K_{2}=-\frac{\left(q_{1} \mathrm{~W}_{1}+q_{2} \mathrm{~W}_{2}\right)}{\sin \Delta}+q_{2} \mathrm{~W}_{2} \cot \frac{\Delta}{2}
\end{aligned}
$$

Let

$$
\lim K_{1}=\lim \frac{\left(q_{1} \mathrm{~W}_{1}+q_{2} \mathbf{W}_{2}\right)}{\sin \Delta}=-\lim K_{2}=K .
$$

Since $K$ is finite, we have at the limit when $\Delta$ tends to $\pi$,

$$
q_{1} \mathbf{W}_{1}+q_{2} W_{2}=0 \text {. }
$$

By putting $K_{1}=K=-K_{2}$ in eqns (5) and (6) and then eliminating $K$ between these equations, we have a new relation free of singularities

$$
y_{1}=\frac{x_{1} \mathbf{S}_{1}\left(1-\mathrm{S}_{1}^{2}\right)}{J\left(1-\mathrm{S}_{1}^{2}-2 J^{2}\right)} \text {. }
$$

Let

$$
n=\frac{r_{1}}{r_{2}}=\frac{q_{2}}{q_{1}}=\frac{1-x_{1}}{1+x_{1}},
$$

be the ratio of the radii. In terms of the optimal thrust angles $\phi_{i}$ and $\psi_{i}$, we first have the condition (26) written as

$$
\phi_{1}=-\phi_{2} \text {. }
$$

Next, we have the condition (29) which becomes

$$
\sin \psi_{1}=-n \sin \psi_{2} \text {. }
$$

To put this equation in a more symmetric form, let

$$
2 \alpha=\psi_{2}-\psi_{1}, \quad 2 \beta=\psi_{2}+\psi_{1} .
$$

Then, equation (33) becomes

$$
(1-n) \tan \alpha=(1+n) \tan \beta .
$$

Finally, after some algebraic manipulation, we can transform eqn (30) into a more explicit form

$$
\begin{aligned}
& y_{1} \cos \beta\left[(n+1)^{2}+4 n\right.\left.\cos ^{2} \beta\right] \\
&=4(n+1)^{2} \tan \phi_{1} \cos \alpha
\end{aligned}
$$

In summary, for a two-impulse nodal transfer, the three necessary conditions for optimality are the eqns (32), (35) and (36). Besides, we must verify that the magnitude of the primer vector does not exceed unity on the three orbits $\mathrm{O}_{1}, \mathrm{O}$ and $\mathrm{O}_{2}$. This condition on the transfer orbit is given by the inequality (9).

\section{THE MINIMIZING NODAL TRANSFER}

There is an infinite number of nodal transfers connecting two orbits $\mathrm{O}_{1}$ and $\mathrm{O}_{2}$ with given elements $e_{i}, p_{i}, \delta_{i}$ and $i$. For distinction, we call the best of these nodal transfers the minimizing nodal transfer and when this nodal transfer also verifies all the Lawden's optimality conditions, we call it the optimal nodal transfer. The minimizing nodal transfer has been studied in[5], and it has been shown that it satisfies the two conditions (32) and (33). Since, in general, the third condition (36) is not satisfied, the minimizing nodal transfer is non optimal. But it is worth analyzing since the cost for this transfer is generally low and the transfer time is finite, of the order of half an orbital period and as such it can be used as a practical transfer between non coplanar elliptical orbits

The line of nodes intersects the first orbit $\mathrm{O}_{1}$ at the points $\mathrm{N}_{1}$ and $\mathrm{N}_{1}^{\prime}$ and the second orbit $\mathrm{O}_{2}$ at the points $\mathrm{N}_{2}$ and $\mathrm{N}_{2}^{\prime}$, where $\mathrm{N}_{i}$ are defined by the true anomalies $\delta_{i}$ and $\mathrm{N}^{\prime}$ by $\delta_{i}+\pi$. It can be shown that the optimal way in connecting these points by a transfer orbit is the same as for the transfer between two equivalent coaxial orbits $\mathrm{O}_{1}^{\prime}$ and $\mathrm{O}_{2}^{\prime}$, in the plane of $\mathrm{O}_{1}$ and $\mathrm{O}_{2}$ respectively, and having the points $\mathrm{N}_{i}$ and $N_{i}^{\prime}$ as apsidal points. Then, we have the following rule[2]: If $e_{1} e_{2} \cos \delta_{1} \cos \delta_{2} \geqslant 0$, the transfer is of the direct, or aligned coaxial type using a transfer orbit connecting the highest apogee with the perigee of the other orbit. If $e_{1} e_{2} \cos \delta_{1} \cos \delta_{2}<0$, we have the inverse coaxial type and the transfer orbit is either between the apogees or between the perigees.

With the positions of the impulses settled, for the aligned coaxial type, we have either $\theta_{1}=\delta_{1}$ and $\theta_{2}=\delta_{2}+\pi$ or $\theta_{1}=\delta_{1}+\pi$ and $\theta_{2}=\delta_{2}$. For the inverse coaxial type we have either $\theta_{1}=\delta_{1}$ and $\theta_{2}=\delta_{2}$ or $\theta_{1}=\delta_{1}+\pi$ and $\theta_{2}=\delta_{2}+\pi$. With the values of $\theta_{1}$ and $\theta_{2}$ well defined, we have the radii $r_{1}$ and $r_{2}$ and also their ratio $n=r_{1} / r_{2}$. The semi-latus rectum of the transfer orbit can be easily evaluated as

$$
p=\frac{2 r_{1} r_{2}}{r_{1}+r_{2}} \text {. }
$$

Before evaluating the characteristic velocities for the transfer, we notice that the rotation $i_{2}$ is of the reflecting type, and hence

$$
i_{1}-i_{2}=i,
$$

with a negative value for $i_{2}$.

Next we explicit the direction cosines of the impulses as given by eqn (20):

$$
\begin{gathered}
\sin \phi_{1}=\frac{u_{1}}{\Delta V_{1}}, \quad \sin \phi_{2}=\frac{u_{2}}{\Delta V_{2}}, \\
\cos \phi_{1} \cos \psi_{1}=\frac{v_{1}}{\Delta V_{1}}, \quad \cos \phi_{2} \cos \psi_{2}=\frac{v_{2}}{\Delta V_{2}}, \\
\cos \phi_{1} \sin \psi_{1}=\frac{w_{1}}{\Delta V_{1}}, \quad \cos \phi_{2} \sin \psi_{2}=\frac{w_{2}}{\Delta V_{2}},
\end{gathered}
$$

with $u_{i}, v_{i}$ and $w_{i}$ given by eqns (17) and (18). To evaluate the rotation angles $i_{1}$ and $i_{2}$ we first use eqn (39) to express the optimal relation (32) as

$$
\frac{\sqrt{v_{1}^{2}+w_{1}^{2}}}{\Delta V_{1}}=\frac{\sqrt{v_{2}^{2}+w_{2}^{2}}}{\Delta V_{2}}, \quad \frac{u_{1}}{\Delta V_{1}}=-\frac{u_{2}}{\Delta V_{2}} \text {. }
$$


On the other hand, we have from the optimal relation (33)

$$
\frac{w_{1}}{w_{2}}=-n \frac{\Delta V_{1}}{\Delta V_{2}}=-n \frac{\sqrt{v_{1}^{2}+w_{1}^{2}}}{\sqrt{v_{2}^{2}+w_{2}^{2}}} .
$$

With the aid of eqns (17) and (18) we explicit this equation as

$$
\frac{\sin i_{1}}{\sin i_{2}}=-n \frac{\left[1+\left(p / p_{1}\right)-2 \sqrt{\left(p / p_{1}\right)} \cos i_{1}\right]^{1 / 2}}{\left[1+\left(p / p_{2}\right)-2 \sqrt{\left(p / p_{2}\right)} \cos i_{2}\right]^{1 / 2}} .
$$

Upon solving eqns (38) and (42), we have the optimal rotations $i_{1}$ and $i_{2}$. From eqn (41), we immediately have the ratio of the characteristic velocities:

$$
\frac{\Delta V_{1}}{\Delta V_{2}}=-\frac{1}{n^{2}} \sqrt{\frac{p_{1}}{p_{2}}} \frac{\sin i_{1}}{\sin i_{2}}=m \text {. }
$$

If we use $\sin f_{2}=-\sin f_{1}$, and substitute eqns (17) and (18) into eqn (39) we have for the other elements

$$
\begin{gathered}
\tan \psi_{1}=\frac{\sin i_{1}}{\sqrt{p / p_{1}}-\cos i_{1}}, \\
\tan \psi_{2}=-\frac{\sin i_{2}}{\sqrt{p / p_{2}}-\cos i_{2}} . \\
\frac{u_{1}}{\sqrt{\mu}}=-\frac{m}{(1+m)}\left(\frac{e_{1}}{\sqrt{p_{1}}} \sin \theta_{1}+\frac{e_{2}}{\sqrt{p_{2}}} \sin \theta_{2}\right), \\
\frac{u_{2}}{\sqrt{\mu}}=\frac{1}{(1+m)}\left(\frac{e_{1}}{\sqrt{p_{1}}} \sin \theta_{1}+\frac{e_{2}}{\sqrt{p_{2}}} \sin \theta_{2}\right) . \\
\tan \phi_{1}=-\frac{m r_{1}\left(\frac{e_{1}}{\sqrt{p_{1}}} \sin \theta_{1}+\frac{e_{2}}{\sqrt{p_{2}}} \sin \theta_{2}\right)}{(1+m) \sqrt{p+p_{1}-2 \sqrt{p p_{1}} \cos i_{1}}} .
\end{gathered}
$$

The characteristic velocities $\Delta V_{i}$ are obtained by eqn (19). They are also explicit functions of the given data $e_{i}, p_{i}, \delta_{i}$ and the computed plane change angles $i_{1}$ and $i_{2}$ which, for each given $i$, depend on the three ratios $n, p / p_{1}$ and $p / p_{2}$.

\section{NUMERICAL EXAMPLES}

The minimizing nodal transfer is valuable only if it is not far from the true optimal transfer. It can be shown that if $C=\Delta V_{1}+\Delta V_{2}$ is the total characteristic velocity for the minimizing nodal transfer and $C^{\prime}$ is the total characteristic velocity for the generalized Hohmann transfer between the equivalent coaxial orbits $\mathrm{O}_{1}^{\prime}$ and $\mathrm{O}_{2}^{\prime}$ with the same plane change $i$, as defined in Section 4, we have the relation

$$
C^{2}=C^{\prime 2}+\left(\frac{h_{1}}{p_{1}} e_{1} \sin \delta_{1}-\frac{h_{2}}{p_{2}} e_{2} \sin \delta_{2}\right)^{2} .
$$

Hence, not only that the transfer time of about half an orbital period is acceptable in practice, the cost for the minimizing nodal transfer under favorable conditions is reasonably close to the cost for an ideal transfer. It is easy to compute the minimizing nodal transfer. After evaluating its elements we can verify the third condition (36). It is trivially satisfied for the coaxial case, $y_{1}=e \sin f_{1}=0, \tan \phi_{1}=0$. In general the condition is not satisfied and the minimizing nodal transfer is not the true optimal transfer between the given terminal orbits.

To assess the usefulness of the minimizing nodal transfer, we consider below two examples. The first one concerns the transfer between two equal orbits and the second example is the transfer from an elliptical orbit to a non coaxial circular orbit. We shall take $\mu=1$ for the computation of the characteristic velocities.

By taking $e_{1}=e_{2}=0.4, p_{1}=p_{2}=1.68$, we have two equal terminal orbits. We assume that the initial orbit $\mathrm{O}_{1}$ is in the equatorial plane. For the terminal orbit $\mathrm{O}_{2}$ besides the prescribed values $e_{2}$ and $p_{2}$, we only require that its perigee is at $90^{\circ}$ before the ascending node, that is $\delta_{2}=90^{\circ}$, and that it is inclined at a certain angle, taken as $i=30^{\circ}$ for the computation. The fact that the longitude of the ascending node of the final orbit $\Omega_{2}=\delta_{1}$ is not important is that the Earth is rotating about an axis orthogonal to the equatorial plane and hence with respect to Earth fixed axes, the line of nodes is automatically adjusted by the Earth's rotation.

Then, we shall use $\delta_{1}$ as parameter and for each value of $\delta_{1}$, from 0 to $180^{\circ}$, we compute the minimizing nodal transfer by the equations in Section 4, and analyze the variation of the total characteristic velocity.

Since $e_{1} e_{2} \cos \delta_{1} \cos \delta_{2}=0$, we have the aligned coaxial type. Since $r_{2}=p_{2}$, when $\delta_{1} \leqslant 90^{\circ}$, the first impulse is applied at $\theta_{1}=\delta_{1}+\pi$, and the second impulse at $\theta_{2}=\delta_{2}$. For $\delta_{1}$ between 90 and $180^{\circ}$, we shall use $\theta_{1}=\delta_{1}$, and $\theta_{2}=\delta_{2}+\pi$. The total characteristic velocity for this transfer is plotted vs $\delta_{1}$ as the upper curve in Fig. 4. In the same figure, we have plotted as the lower curve the total characteristic for the optimal two-impulse transfer, using the optimal switching relations in Section 2. The two curves exhibit symmetry with respect to the line $\delta_{1}=90^{\circ}$. The usual deviation between these two curves is about $10 \%$ and reduces to $6 \%$ when they are near their minimum values which occur for a nearly same

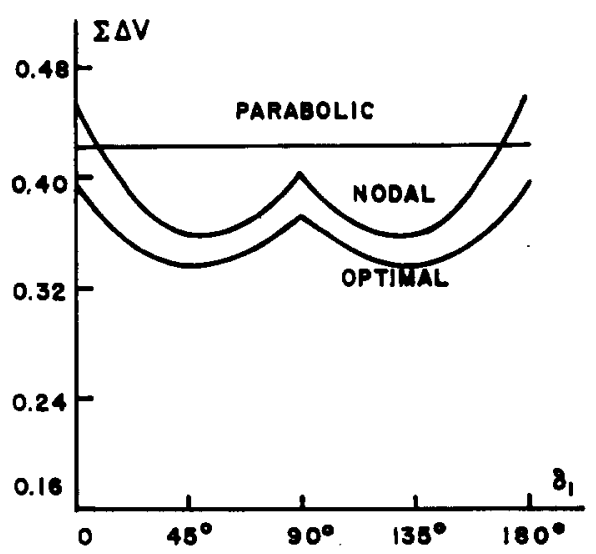

Fig. 4. Characteristic velocities for Case 1 . 


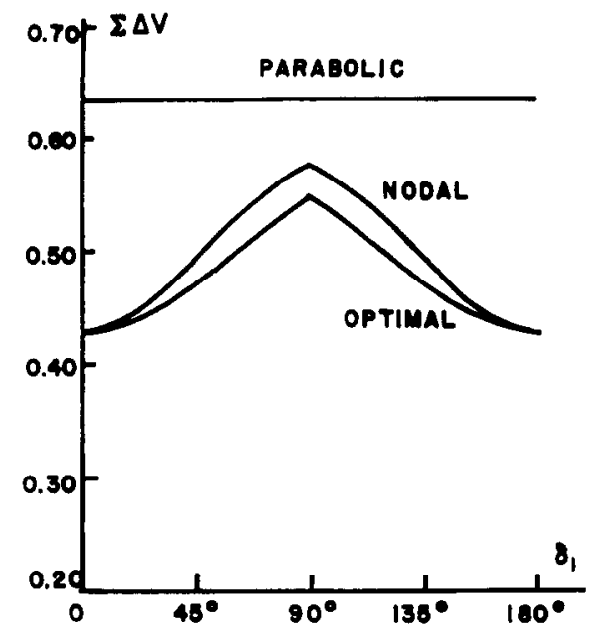

Fig. 5. Characteristic velocities for Case 2 .

value of $\delta_{1}$. Hence, for a transfer from $\mathrm{O}_{1}$ to $\mathrm{O}_{2}$ when $\delta_{1}=\Omega_{2}$ is not specified, we can use the minimizing nodal transfer to quickly determine the approximate optimum value $\Omega_{2}^{*}$ before calculating the transfer. As a check of the optimality of the solution we have plotted in Fig. 4 the constant cost for a transfer via parabolic orbits which is

$$
\Delta V_{\mathrm{P}}=\sum \sqrt{\frac{\mu}{p_{i}}}\left[\sqrt{2\left(1+e_{i}\right)}-\left(1+e_{i}\right)\right] .
$$

In the present case, $\Delta V_{\mathrm{P}}=0.4217$. It is seen that the cost for the minimizing nodal transfer is sufficiently close to the optimal value for it to be a useful transfer.

In the second example, we use the same initial orbit $\mathrm{O}_{1}$ in the equatorial plane, with the same plane change $i=30^{\circ}$. This time we take the final orbit as circular with $e_{2}=0, p_{2}=1$. Again we use the longitude of the ascending node $\delta_{1}=\Omega_{2}$ as a parameter varying from 0 to $180^{\circ}$, while $\delta_{2}$ is of course arbitrary. This is a practical configuration usually encountered when we transfer from an elliptical orbit in the equatorial plane to an inclined circular orbit at low altitude. Again, the transfer is of the aligned coaxial type and since $\mathrm{O}_{2}$ is completely inside $\mathrm{O}_{1}$, we use the same selection for $\theta_{1}$ and $\theta_{2}$ as in the first case. The characteristic velocities are plotted in Fig. 5 and we have a deviation of about $6 \%$. When $\delta_{1}=0$ and $180^{\circ}$, the two orbits are coaxial and the minimizing nodal transfer is also the optimal transfer. In this case, the cost for the parabolic transfer as computed from eqn (48) is $\Delta V_{\mathrm{P}}=0.6251$ and is non optimal.

\section{CONCLUSION}

In this paper, we have presented the necessary conditions for the minimum fuel, time-free transfer between two non-coplanar elliptical orbits. It is shown that the solution is obtained by solving a system of three non-linear equations for three unknowns. It is then the best two-impulse transfer but if some additional inequalities are not satisfied, the three-impulse transfer may be optimal.

In the second part we discuss the case where the impulses are applied along the line of nodes. In general, this nodal transfer is non optimal, but the best nodal transfer, called the minimizing nodal transfer is reasonably close to the optimal transfer to be useful as its substitute for a preliminary evaluation of the fuel consumption. Furthermore, when we continuously vary the relative position of the terminal orbits, the variations of the two total characteristic velocities exhibit the same trend in the sense that they pass through their minima at nearly the same relative position. This makes the set of explicit formulae for computing the minimizing nodal transfer, as presented in this paper, a useful tool for designing a minimum fuel transfer between several orbits. For lack of space we plan to discuss the special case of one impulse transfer and the non optimality of the noncoaxial three-impulse nodal transfer in a separate paper.

Acknowledgement-This work was supported by the Jet Propulsion Laboratory under contract No. 956416 with Dr K. D. Mease as project manager

\section{REFERENCES}

1. D. F. Lawden, Optimal Trajectories for Space Navigation. Butterworths, London (1963).

2. J. P. Marec, Optimal Space Trajectories. Elsevier, Amsterdam (1979)

3. C. Marchal, Une expression des commutations sous forme explicite dans le problème des transferts optimaux entre orbites képlériennes. Rech. Aerosp. 1971, 1-7 (1971).

4. K. G. Eckel and N. X. Vinh, Optimal switching conditions for minimum fuel fixed time transfer between non-coplanar elliptical orbits. Acta Astronautica 11, $621-631$ (1984).

5. F. T. Sun, Analytic solution for optimal two-impulse $180^{\circ}$ transfer between noncoplanar orbits and the optimal orientation of the transfer plane. AIAA J. 7 , 1898-1904 (1969). 